\title{
Tempting long-memory - on the interpretation of DFA results
}

\author{
D. Maraun ${ }^{1}$, H. W. Rust ${ }^{2}$, and J. Timmer ${ }^{3}$ \\ ${ }^{1}$ Department of Physics, Potsdam University, D-14415 Potsdam, Germany \\ 2 Potsdam Institute for Climate Impact Research, P.O. Box 6012 03, D-14412 Potsdam, Germany \\ ${ }^{3}$ Center for Data Analysis and Modeling, Albert-Ludwigs Universität, D-79104 Freiburg, Germany
}

Received: 20 August 2004 - Revised: 26 October 2004 - Accepted: 8 November 2004 - Published: 10 November 2004

Part of Special Issue "Nonlinear analysis of multivariate geoscientific data - advanced methods, theory and application"

\begin{abstract}
We study the inference of long-range correlations by means of Detrended Fluctuation Analysis (DFA) and argue that power-law scaling of the fluctuation function and thus long-memory may not be assumed a priori but have to be established. This requires the investigation of the local slopes. We account for the variability characteristic for stochastic processes by calculating empirical confidence regions. Comparing a long-memory with a short-memory model shows that the inference of long-range correlations from a finite amount of data by means of DFA is not specific. We remark that scaling cannot be concluded from a straight line fit to the fluctuation function in a log-log representation. Furthermore, we show that a local slope larger than $\alpha=0.5$ for large scales does not necessarily imply longmemory. We also demonstrate, that it is not valid to conclude from a finite scaling region of the fluctuation function to an equivalent scaling region of the autocorrelation function. Finally, we review DFA results for the Prague temperature data set and show that long-range correlations cannot not be concluded unambiguously.
\end{abstract}

\section{Introduction}

Understanding the variability of the climate system on scales from days to decades or even longer is a fundamental issue of climatology. In 1976, Hasselmann introduced the concept of stochastic climate models and showed that climate variability in principle can be modeled by rather simple ARprocesses. The time dependency of linear stochastic models is completely captured by the autocorrelation function $C(s)$, describing the average linear relation of two points in time with lag $s$. The sum of $C(s)$ over all lags $s$ is often called memory or persistence of the process (Trenberth, 1985). Typical properties of AR-processes are an exponential decay of the autocorrelation function for large $s$ and thus

Correspondence to: D. Maraun

(maraun@agnld.uni-potsdam.de) a finite memory which is equal to the existence of a characteristic time scale. Processes with these attributes are called short-range correlated or short-memory processes.

Later, Pelletier and Turcotte (1999) as well as KoscielnyBunde et al. (1998) reported long-range correlations in temperature data. This process property is characterized by infinite memory and the absence of a typical time scale. For large time lags $s$, the autocorrelation function of such long-memory processes decays according to a power-law and hence exhibits scaling with a characteristic exponent $\gamma$.

Govindan et al. (2002) reported, that widely used general coupled climate models do not exhibit the behavior found by Koscielny-Bunde et al. (1998) and raised the question whether estimates of the warming-trend in global mean temperature based on these models could be exaggerated. However, Fraedrich and Blender (2003) extended the analysis to various stations around the globe and showed that state-ofthe-art climate models are able to reproduce the empirical findings.

Except for Pelletier, the authors applied Detrended Fluctuation Analysis (DFA, Peng et al., 1993) which investigates the average variability on a scale $s$. For long-memory processes in the limit of large scales, this fluctuation function $F(s)$ can directly be linked to the autocorrelation function. However, in all works known to the authors the properties of DFA have only been studied for certain long-memory processes. The behavior for other linear processes has not been investigated and it is not clear if the results can be easily transferred.

In this paper we interpret the inference of long-memory from empirical data as an inverse problem. The main insight is, that power-law scaling of the fluctuation function and thus long-memory may not be assumed a priori but have to be established. We argue that this requires the investigation of the local slopes of the fluctuation function. Furthermore one has to account for the variability characteristic for stochastic processes. Associated with the inference of long-range correlations, we investigate the sensitivity and specificity of DFA: When long-memory is present, does DFA detect it? When 
no long-memory is present, does DFA reject it? We illustrate our discussion by comparing a long-memory with a shortmemory model. Finally, we review the results of KoscielnyBunde et al. (1998) for the Prague temperature data set and show that their conclusions are not unambiguous.

In Sect. 2, we introduce the processes considered and discuss the inference of long-range correlations. The method of DFA is explained in Sect. 3. In Sect. 4, we interprete the inference of long-range correlations from empirical data as an inverse problem and present the associated terms and questions. To exemplify the discussion, we present two models in Sect. 5. Establishing power-law scaling of the fluctuation function is discussed with respect to local slopes and natural variability in Sect. 6. Section 7 gives an overview over typical pitfalls. As a consequence of the foregoing discussions, we review the DFA results of the Prague daily temperature record in the last Section.

\section{Short- and long-memory}

Many processes in nature are of such a high complexity that a description by deterministic models is difficult or not desirable. However, their characteristic behavior often is effectively captured by stochastic processes. In 1976, Hasselmann introduced the concept of stochastic climate models and showed that the variability of the climate system in principle can be modeled by rather simple and linear ARprocesses. The time dependent structure of linear stochastic processes is captured by the autocorrelation function $C(s)$ of two points in time with lag $s$. The integral of $C(s)$ over all lags $s$ is often called memory or persistence (Trenberth, 1985).

Different classes of autocorrelation structures can be distinguished with respect to the form of their decay for large time lags $s$ : Many stochastic processes in nature exhibit short-range correlations, which decay exponentially:

$C(s) \propto e^{-s / \tau}$, for $s \rightarrow \infty$.

These processes exhibit a typical time scale $\tau$. This decay is fast enough to let the sum $\sum C(s)$ converge resulting in a finite decorrelation time (von Storch and Zwiers, 1999), e.g. for $C(s)=e^{-s / \tau}$

$$
\begin{aligned}
\tau_{D} & =1+2 \sum_{s=1}^{\infty} C(s)=1+2 \sum_{s=1}^{\infty} e^{-s / \tau} \\
& \approx 2 \tau \text { for } \tau \gg 1 .
\end{aligned}
$$

We employ the decorrelation time as a measure for the memory or persistence of a process. Correlations on scales large compared to the decorrelation time are negligible due to the fast exponential decay. Thus, one also refers to these processes as having short-range or finite memory. Typical examples are AR-processes.

A second class are long-range correlated processes characterized by algebraically decaying correlations:

$C(s) \propto s^{-\gamma}$, for $s \rightarrow \infty, \quad$ with $0<\gamma<1$.
A characteristic time scale as defined above does not exist The calculation of the decorrelation time, e.g. for $C(s)=s^{-\gamma}$ for $s>0, C(0)=1$

$\tau_{D}=1+2 \sum_{s=1}^{\infty} C(s)=1+2 \sum_{s=1}^{\infty} s^{-\gamma}=\infty$

results in infinite memory. A physical example for such a process can be found in Kolmogorov's theory of turbulence (Frisch, 1995). A mathematical model exhibiting long-range correlation is e.g. fractional Gaussian noise (FGN) (Mandelbrot and van Ness, 1968). For this process the asymptotic relation Eq. (3) is already well satisfied for finite sample sizes because it is the increment of the self-similar process fractional Brownian motion. A more flexible long-range correlated process is given by fractional $\operatorname{ARIMA}[p, d, q]$ (FARIMA) (Beran, 1994; Granger, 1980; Hoskins, 1981). However, for non-trivial autoregressive (AR) or moving average (MA) components $(p+q>0)$ it is possible that the asymptotic relation Eq. (3) is not satisfied for a finite sample.

The concept of long-memory refers to non-periodic processes. Thus, the recurrence due to periodicities like the Milankovitch-cycles in the climate system are not to be considered as long-range correlations, even if their (deterministic) behavior causes correlations for infinite time lags.

An important question to characterize a given non-periodic process is now to investigate, if its autocorrelation decays exponentially or according to a power law. However, for observational data, one cannot investigate the decay of the autocorrelation function on arbitrarily large scales. Measurement limitations always restrict the analysis and interpretation to a range between two time scales independent of the nature of the process: First, the sampling interval $\Delta t$ defines a minimum time scale. Second, the finite length of the record $T$ defines a maximum time scale. Intuitively, one might easily be tempted to refer to records with characteristic time scales close to $\Delta t$ as short-range and close to $T$ as long-range correlated. This, however, ignores the fundamental difference of short-range and long-range correlated processes concerning the form of the decay of their autocorrelation as given in Eqs. (1) and (3). This difference is essential e.g. for estimation of the variance of the mean, prediction (Beran, 1994), trend assessment (Kallache et al., 2004 ${ }^{1}$; Giratis, et al., 2001) and extreme value statistics (Elek and Márkus, 2004).

\section{Detrended Fluctuation Analysis (DFA)}

Estimating the autocorrelation function $C(s)$ from empirical data is limited to rather small time lags $s$ and is affected by observational noise and instationarities like trends. Peng et al. (1994) suggested Detrended Fluctuation Analysis (DFA) to indirectly gain information about the correlation

\footnotetext{
${ }^{1}$ Kallache, M., Rust, H., and Kropp, J.: Trend Assessment: Applications for Hydrology and Climate Research., Nonlinear Proc. Geophys., submitted, 2004.
} 
structure imposed on a time series. The method works as follows: Given a realization $x(i)$ of length $N$ of a stochastic process, one calculates the profile $y(j)$ :

$y(j)=\sum_{i=1}^{j}[x(i)-\bar{x}], \quad$ with $\quad \bar{x}=\frac{1}{N} \sum_{i=1}^{N} x(i)$.

The profile $y(i)$ is divided into $M$ non-overlapping segments of length $s$. For DFA $n$, in each segment $m$ a best fit polynomial trend $p_{s, m}^{n}$ of order $n$ is subtracted from the profile:

$Y_{s, m}(i)=y(i)-p_{s, m}^{n}(i)$.

For each segment $m$ the squared fluctuation is calculated:

$F_{m}^{2}(s)=\frac{1}{s} \sum_{i=(m-1) s+1}^{m s} Y_{i}^{2}$

The squared fluctuation function of the process is estimated by averaging over all segments:

$F^{2}(s)=\frac{1}{M} \sum_{m=1}^{M} F_{m}^{2}(s)$

This procedure is repeated for several $s$. The minimum scale is given by the sampling interval $\Delta t$ and the order $n$ of DFA, a reasonable choice for the maximum scale is about $1 / 10$ of the total record length, due to natural variability as will be discussed in Sect. 6. For a more comprehensive description of the algorithm and the influence of trends refer to Kantelhardt et al. (2001).

For an uncorrelated series $x(i)$ we get a squared fluctuation function $F^{2}(s) \propto s$ (i.e. $F(s) \propto s^{0.5}$ ) which reflects the linear increase of the variance of the profile. For time series $x(i)$ with algebraically decaying autocorrelations (Eq. 3), it can be shown that in the limit of large $s$ the fluctuation function increases according to a power law (Taqqu et al., 1995)

$F(s) \propto s^{\alpha}, \quad \alpha=1-\gamma / 2, \quad$ with $0.5<\alpha<1$.

Thus, analyzing the fluctuation function of long-range correlated processes for large $s$ reveals the decay exponent of the corresponding correlation function for large $s$.

Taqqu et al. (1995) have shown that DFA (with a subsequent straight line fit in the log-log plot) is an effective estimator for the Hurst coefficient $H$ or equivalently for the exponent $\gamma=2-2 H$ describing the power-law decay of the autocorrelation function. The ability of DFA to estimate $\alpha$ from realizations of long-memory processes corrupted by different instationarities like trends, spikes, harmonics and gaps has been studied by $\mathrm{Hu}$ et al. (2001), Chen et al. (2002) and Kantelhardt et al. (2001).

\section{The inverse problem}

In time series analysis, one aims to infer properties of an underlying process, such as the process class itself or its parameters, from observational records. This is referred to as an inverse problem (Honerkamp, 1998). If the class of the process is not known a priori, it first has to be identified. This is exactly the setting when inferring the correlation structure from realizations of an unknown process: Given the set of all possible correlation structures, the inference of long-range correlations means

1. showing the compatibility of the given data set with a realization of a long-memory process,

2. excluding other possible correlation structures.

These two conditions - the necessary and the sufficient - are often discussed with respect to the terms of sensitivity and specificity: A procedure, that with a high probability detects compatibility with long-range correlations, whensoever they are present, is called sensitive. An algorithm that with a high probability rejects long-range correlations, when they are not present, is said to be specific. The optimal algorithm would be sensitive and specific. A sensitive but unspecific algorithm, however, would produce many false positive results, i.e. one would frequently detect long-range correlations. This algorithm would not be suitable for a reliable inference. On the other hand, an un-sensitive but specific algorithm would be very conservative and would often reject the existence of long-range correlations.

To our knowledge it has not been studied, if DFA can be used to infer long-memory from realizations of a process when it is not a priori clear, if this process is long-range correlated. I.e. it is still an open question, how sensitive and specific DFA behaves when investigating processes of unknown correlation structure for long memory.

A necessary condition for the existence of long-range correlations is the scaling of the fluctuation function $F(s)$ according to Eq. (9). Thus, in this paper, we mainly address the following questions:

1. How to conclude scaling from the DFA fluctuation function?

2. Does scaling necessarily imply long-range correlations?

\section{Two example processes}

To illustrate our line of argumentation, we consider a shortmemory as well as a long-memory process and apply DFA to both. The choice of the model parameters is motivated by the example of the Prague temperature record studied in Sect. 8 and will become clear during the discussion. As an example for a long-range correlated process we simulate fractional Gaussian noise according to the method given in Timmer and König (1995) with $\alpha=0.6$. This process shows power-law scaling in the autocorrelation function for a wide range of scales. For the short-range correlated process we choose a superposition of three AR[1]-processes,

$x(i)=\sum_{j=1}^{3} A_{j} y_{j}(i), \quad y_{j}(i)=a_{j} y_{j}(i-1)+\eta_{j}(i)$, 


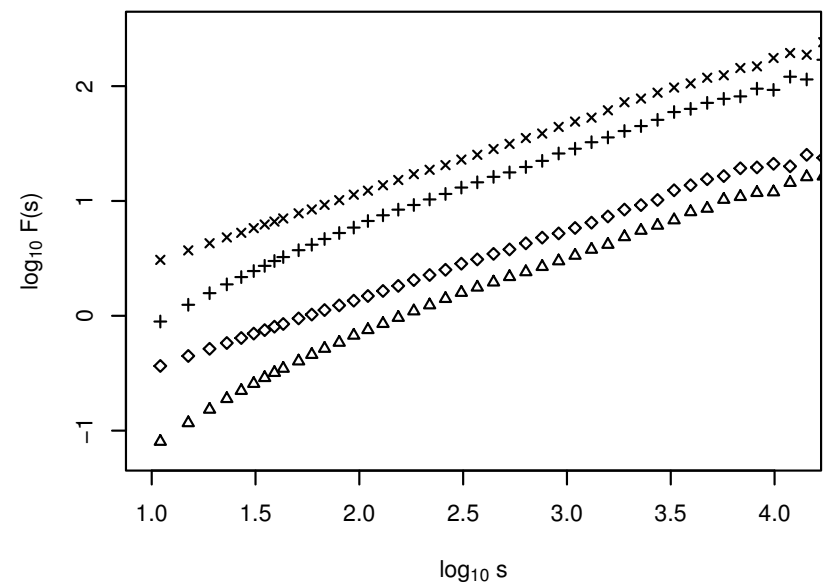

Fig. 1. Fluctuation functions calculated for an artificial long-range correlated process with exponent $\alpha=0.6(\times$ DFA1, $\diamond$ DFA2) and a superposition of three AR-processes ( + DFA1, $\triangle$ DFA2) as defined in Sect. 5. For each order of magnitude, approx. 50 values are calculated. To enhance clarity, only every third value is plotted.

with $\eta_{j}(i)$ being Gaussian white noise of zero mean and variance $1-a_{j}^{2}$. The latter insures var $\left(y_{j}\right)=1$. We choose $A_{1}=0.913, A_{2}=0.396, A_{3}=0.098, a_{1}=0.717, a_{2}=0.953$ and $a_{3}=0.998$. Using $a_{j}=e^{-1 / \tau_{j}}$ we find the following characteristic time scales for the individual AR[1] processes: $\tau_{1}=3 \mathrm{~d}$, $\tau_{2}=21 \mathrm{~d}$ and $\tau_{3} \approx 1.5$ years.

\section{Establish scaling}

Figure 1 shows the fluctuation functions for a realization of each of the two example processes defined in the previous section with $N=70492$ and $\Delta t=1$. For each order of magnitude 50 values of equal distance in logarithmic scale are calculated. For clarity reasons, we plotted only every third value.

To reliably infer power-law scaling of the fluctuation function, a straight line in the log-log plot has to be established. Since a straight line is tantamount to a constant slope, the local slopes $\alpha(s)$ of $\log _{10} F(s)$ vs. $\log _{10} s$ have to be evaluated for constancy in a sufficient range (Kantz and Schreiber, 1995; Tsonis and Elsner, 1995; Timmer et al., 2000). The extend of a sufficient range is still a matter of debate (see e.g. Avnir et al. (1998) and references therein). This concept has been introduced in the context of estimating correlation dimensions (Caputo et al., 1986; Tsonis and Elsner, 1995) and, in a different setting, has also been suggested for DFA (Peng et al., 1993).

For a finite amount of data the estimation of the local slopes brings along a certain variability and even for a longmemory process like FGN, the local slopes of the empirical fluctuation function show variations around a constant $\alpha$. This has two consequences for the calculation and interpretation of the local slopes: First, estimating the local slopes by finite differences results in a large variability. This can be reduced fitting a straight line to $\log (F(s))$ vs. $\log (s)$ within a

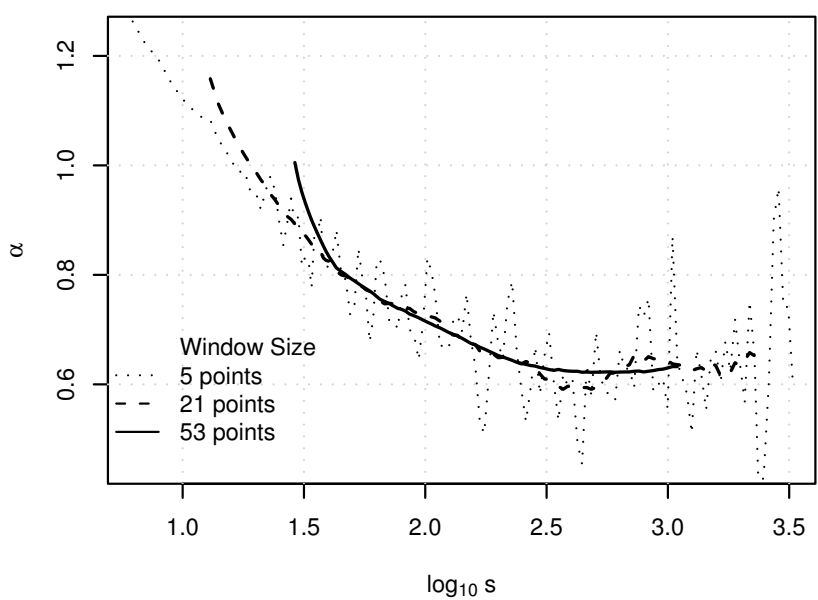

Fig. 2. Local slopes of a realization of the short-memory model for different window sizes. For $F(s)$, approx. 50 points per order of magnitude are calculated. For small windows, the bias is very low, but the variability renders the interpretation difficult, whereas for large windows, the variance is reduced at the cost of a biased estimate of $\alpha$.

small window. The window is then shifted successively over all calculated scales $s$. Figure 2 shows the local slopes of a realization of the short-memory model for different window sizes. Choosing the optimal window size, one has to trade bias for variance: For small windows, the bias is small, but the variability renders the interpretation difficult, whereas for large windows, the variance is reduced at the cost of a biased estimate of $\alpha$. Thus, the extreme case of a single straight line fit to the whole range of scales considered is maximally biased. Since only one value of $\alpha$ is calculated, this does not allow to evaluate constancy.

As a second consequence of the finite amount of data, one has to quantify the variability for a given length of the record. Since vicinal local slopes are not independent, confidence regions cannot be estimated easily from the procedure described in Sect. 6 (Denker and Keller, 1986). Instead, we perform Monte Carlo simulations: For the two example processes, we simulate 1000 realizations to estimate mean and standard deviation of $\alpha$ for the scales considered. For a fixed scale $s$, the distribution of $\alpha(s)$ is approximately gaussian. Thus, we employ $\bar{\alpha}(s) \pm 1.96 \sigma(s)$ as estimates of the $95 \%$ confidence bands.

Figure 3 displays the local slopes of the DFA1 (a) and DFA2 (b) fluctuation functions, estimated from one realization of each of the example models using a window of 21 points. Additionally, the corresponding $1.96 \sigma$ intervals around the mean of each model are plotted. The realization of the long-memory process shows fluctuations around a constant $\alpha$ within the corresponding $1.96 \sigma$ interval, increasing like $\sigma \propto \sqrt{s}$ (Peng et al., 1993). The local slope $\alpha(s)$ of the short-memory realization, however, decreases constantly in the beginning and basically follows the local slope of the long-memory realization for scales larger than $\log _{10} s \approx 2.5$. Thus, for a certain choice of parameters, a short-range corre- 
lated model can mimic scaling in a finite range. Due to the principle of variance superposition for DFA (Hu et al., 2001), a suitable superposition of three AR[1] processes produces this effect in the fluctuation function analogously to the same effect in the spectral domain described in Hausdorff and Peng (1996).

Analyzing the long-memory properties one studies primarily the behavior on large scales $s$ assuming that influences from short-range components are negligible and do not bias the estimation of the long-range dependence parameter. In our example, the $1.96 \sigma$-cones are virtually indistinguishable in this range. Thus, based on the given record length and only considering large $s$, one cannot distinguish the realizations of the two models by means of DFA. For longer time series, the cones would shrink and the region of overlapping would become smaller.

However, a general dilemma related to the inference of long-memory emerges: For a finite time series, one will always find a short-range correlated model to describe the data (Beran, 1994). Thus, considering the inference of longmemory, DFA is sensitive, but not specific. An alternative is to investigate if the underlying process is short-range correlated. Transferring the discussion in Sect. 4, this requires:

1. To show compatibility with a short-range correlated model.

\section{To exclude possible long-range correlated models.}

The first condition is always fulfilled, since one will always find a short-range correlated model to describe a finite data set. Thus, for the inference of short-memory, DFA is sensitive. The second condition is not fulfilled for the given example, because the record length is not sufficient to detect the short-memory character $\alpha=0.5$ for large $s$ of the AR-model by means of DFA. For longer time series as shown in Fig. 4, when a plateau of $\alpha=0.5$ is identifiable, long-memory can be excluded and the specificity of DFA to infer short-range correlations increases.

The capability of short-memory models to reproduce findings which are associated with long-memory has also been considered with respect to the Hurst phenomenon (McLeod and Hipel, 1978; Montanari et al., 2000). However, it is not always meaningful to model finite data with a short-memory model: the longer the scaling region of a short-range model shall be, the more parameters and tuning are required. It may be advantageous to describe a data set exihibiting several orders of magnitude of power-law scaling with a longrange correlated model with few parameters rather than with a short-range correlated model with a large number of parameters. To decide which model to prefer with respect to parameter parsimony, one could e.g. employ a likelihood approach combined with an Akaike-type model selection criterion (Beran et al., 1998).

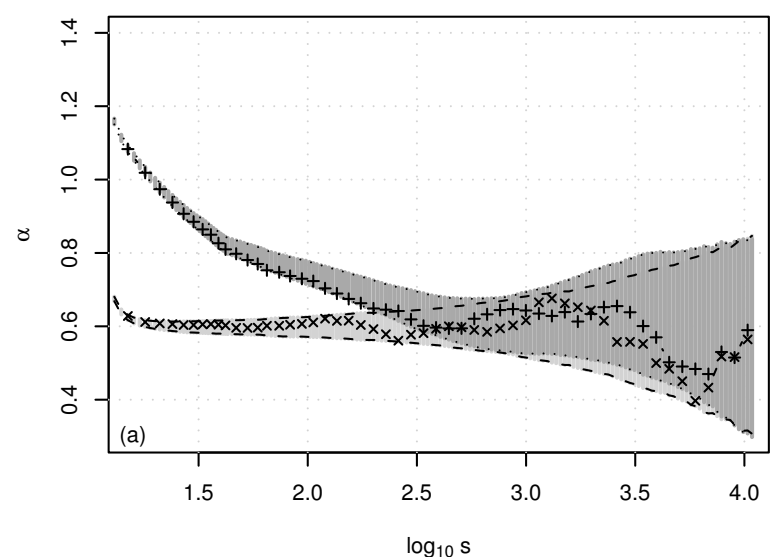

(a)

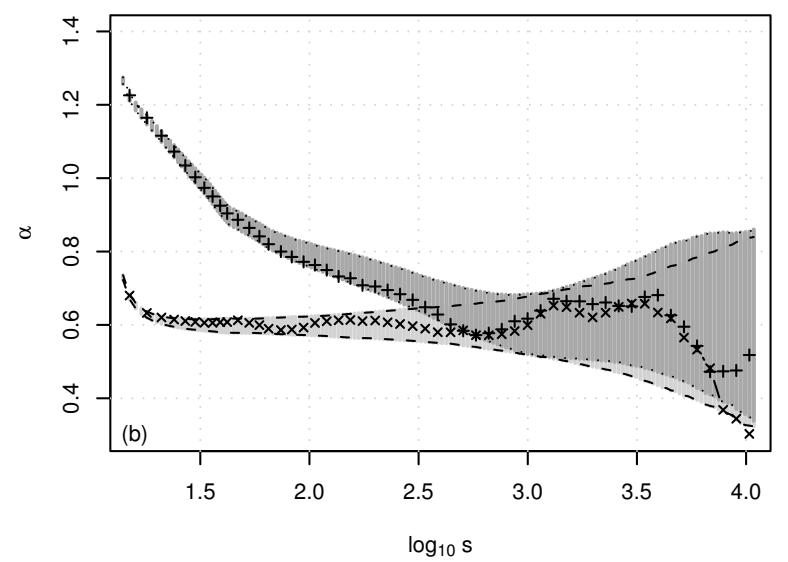

(b)

Fig. 3. Local slopes of the (a) DFA1 and (b) DFA2 fluctuation function calculated for an artificial long-range correlated process with exponent $\alpha=0.6(\times)$ and a superposition of three AR-processes $(+)$ as defined in Sect. 5. The dashed and the dotted lines border the shadowed $1.96 \sigma$ intervals obtained from 1000 realizations of the two processes, respectively.

\section{Pitfalls}

\subsection{The double logarithmic plot}

Investigating only the double logarithmic plot of the fluctuation function, one is tempted to rashly conclude for longrange correlations. Due to properties of the logarithm, fluctuations are suppressed in a log-log plot and the deviation from a straight line is not easily visible (Tsonis and Elsner, 1995). Also, restricting the analysis to a straight line in the log-log plot forces $F(s)$ in the procrustean bed of power-laws. It will always yield some value for the slope but the suitability of the linear description is not evaluated. For the inference of longrange correlations, this procedure would be sensitive but not specific in the sense, that long range correlations would be attributed to all processes with $\alpha \neq 0.5$ for the largest scale observed. Such a result would trivialize the concept of longrange correlations and provide no insight into the process. Thus, to reliably infer a power-law, a straight line may not be assumed a priori but has to be established, as discussed 


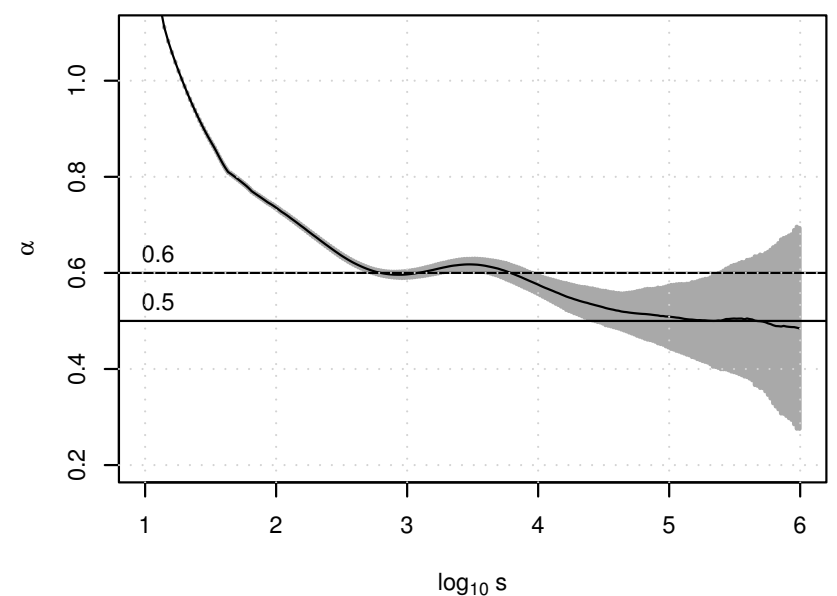

Fig. 4. Empirical fluctuation function of the short-memory model Eq. (10), estimated from 200 realizations of length $N=1000000$ (solid line). A region of approximatively constant slope occurs between $\log _{10} s \approx 2.8$ ( $\left.s \approx 600\right)$ and $\log _{10} s \approx 3.8$ ( $s \approx 6000, \simeq 16$ years). On larger scales, the slope reduces to $\alpha=0.5$ characterizing the short-memory nature of the model.

in Sect. 6. Even if scaling is present, it is difficult to determine the beginning and ending of the scaling region in the $\log -\log$ plot. However, the resulting value for $\alpha$ derived from a straight line fit strongly depends on the fit boundaries if the realization does not stem from a scale free process.

\subsection{Finite scaling of short-memory processes}

According to Sect. 2, the autocorrelations of short memory processes decay exponentially for large $s$ and are negligible on scales large compared to the decorrelation time $\tau_{D}$ (Eq. 2). Consequently, for scales large enough, the slope of the fluctuation function of such a process converges to $\alpha=0.5$. However, for a finite set of data one cannot be a priori sure that the series is long enough to observe this. For a record of the short-memory model definded in Sect. 5 of length 70492 points the local slopes of the fluctuation function of the largest observed scales is compatible with powerlaw scaling. A plateau with $\alpha=0.5$ is not observed (Fig. 3). Thus, one might be tempted to conclude long-memory. However, analysing a much longer record (1 000000 points) of the same model yields a plateau with $\alpha=0.5$ for large $s$ as can be seem from Fig. 4. Therefore, for a process with unknown correlation structure it is misleading to use $\alpha>0.5$ as evidence for long-memory. It might very well be that the record is too short to observe a plateau with $\alpha=0.5$.

\subsection{Shift of the finite scaling region}

As shown in Sect. 6, under certain conditions also shortmemory processes can exhibit a finite "scaling" region. Thus, the question arises, if such a scaling region derived from the fluctuation function corresponds to the same region in the auto correlation function. To address this question, we relate the fluctuation function shown in Fig. 4 to the analyti-

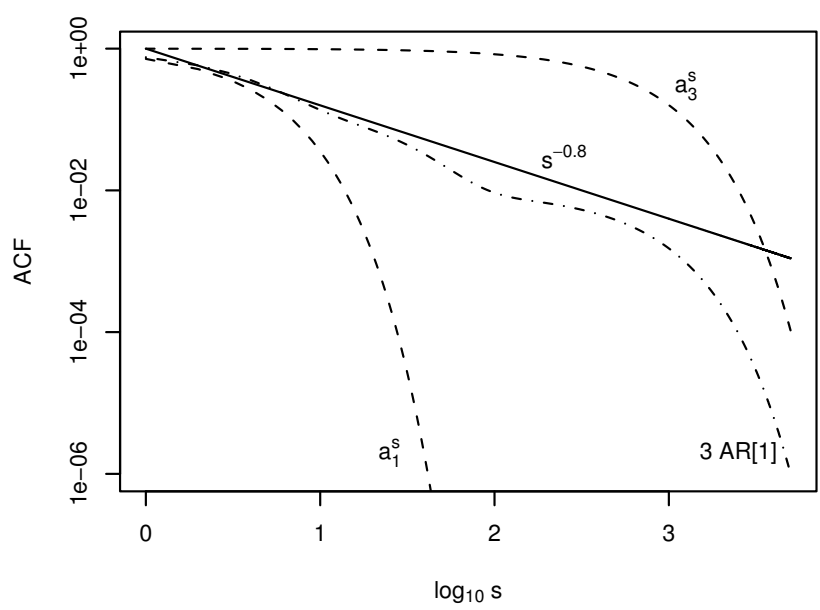

Fig. 5. Analytical autocorrelation function of the short-memory model Eq. (10) (dashed dotted line). The dashed lines depict the autocorrelation functions of the single AR[1] processes with shortest and longest time constant respectively. In a scale range from $\log _{10} s \approx 0(s \approx 1)$ to maximally $\log _{10} s \approx 3(s \approx 1000)$ the autocorrelation function approximately follows the power law with $\gamma=0.8$ (solid line). For larger scales, it turns into an exponential decay determined by the AR[1] process with the largest time constant $\tau \approx 1.5$ years.

cal autocorrelation function as shown in Fig. 5. The dashed lines depict the autocorrelation functions of the single AR[1] processes with the largest and the smallest time scale, the autocorrelation function of the superposition of the three AR[1] processes is given by the dashed-dotted line. The solid line represents a power-law with exponent $\gamma=0.8$ as expected, when applying Eq. (9) to the exponent $\alpha=0.6$ as derived from the fluctuation function. We find that the region of almost constant slope of the autocorrelation function is located on smaller scales between $s \approx 1$ and maximally $s \approx 1000(\simeq 3$ years). Thus, based on a finite scaling region found in the fluctuation function of a short-memory process, it is not valid to conclude that an equal scaling region exists also for the autocorrelation function.

\section{Memory of temperature records}

As a consequence of the previous discussion, we review a prominent DFA result. It has been stated by KoscielnyBunde et al. (1998), that temperature records follow a universal scaling law. This behavior was said to be found in numerous data sets. For a discussion of the existence of universal scaling exponents see Tsonis et al. (2000). Here, we want to check these findings exemplarily in the daily air temperature dataset of Prague starting from 1 January 1800 up to 12 December $1992(N=70492)$. To test, whether the underlying process is long-range or short-range correlated, we adjusted the long-range correlated model and the AR-model given in Sect. 5 to the data set.

We apply DFA1 and DFA2 to the temperature anomalies $\Delta T_{i}=T_{i}-\bar{T}_{i}$ giving the deviation of a days temperature from 


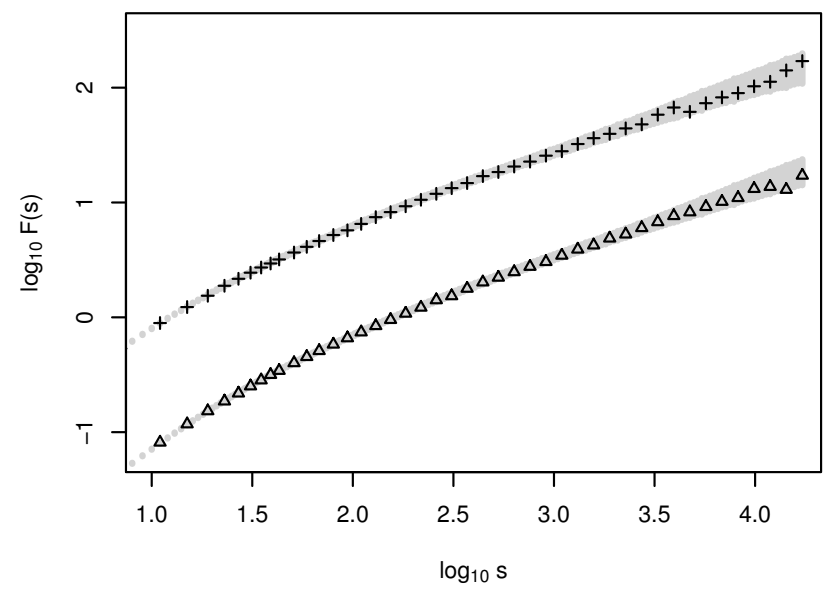

Fig. 6. DFA1 $(+)$ and DFA2 $(\triangle)$ fluctuation function of Prague daily air temperature data caculated for approximately 50 points per order of magnitude. Only every 4th point is shown to enhanced clarity. The shadows mark the $1.96 \sigma$ confidence regions derived from 1000 runs of the AR model.

the average over several years for this day of the year. An investigation of higher orders of DFA does not significantly affect the discussion presented while for DFA1 the effect of a trend might be suspected. $F(s)$ is calculated for approximately 50 points per order of magnitude upto $s_{\max }=N / 4$ and is shown in double logarithmic representation in Fig. 6 for DFA1 and DFA2.

The behavior is qualitatively different from white noise. However, following the discussion in Sect. 6, we have to estimate the local slopes to investigate for power-law scaling. From the fluctuation function, we estimate the local slopes using a straight line fit in a small window of 21 points.

According to Sect. 6, DFA is not specific when investigating for long-memory. However, we alternatively study if long-memory can be excluded. Figures $7 \mathrm{a}$ and $7 \mathrm{~b}$ show the local slopes for DFA1 and DFA2 of the Prague daily temperature record and additionally the $1.96 \sigma$ confidence intervals derived from the two models. For both orders of DFA the result for the empirical data is almost completely within the $1.96 \sigma$ interval of the short-memory process. In the range of large $s$ with high variability, we find the local slopes also within the $1.96 \sigma$ interval corresponding to the long-memory process. Thus, from the given data, one cannot decide whether the Prague temperature time series is a realization of a short-memory or a long-memory process. However, considering all scales observed, the short- memory model describes the data better than the long-memory model with respect to DFA. If the underlying process was shortrange correlated, data sets of sufficient length would provide estimates of sufficient accuracy to identify the short-memory character. According to Sect. 7.2, we expect such a time series to be much longer as the recorded ones.

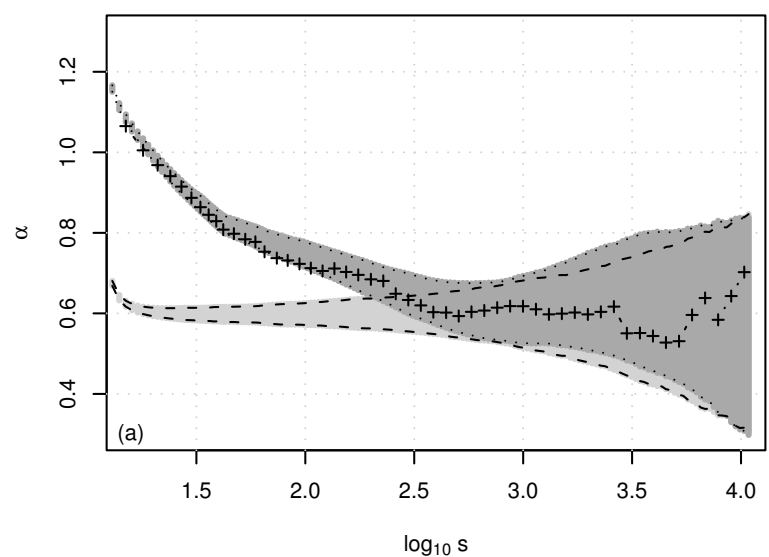

(a)

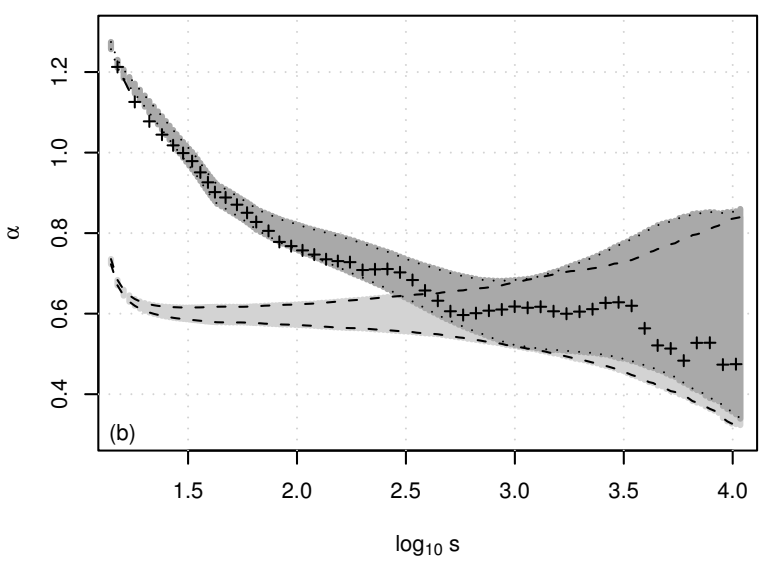

(b)

Fig. 7. Local slopes of the fluctuation functions plotted in Fig. 6 for (a) DFA1 and (b) DFA 2 of the Prague daily temperature data. The dotted lines border the $1.96 \sigma$ confidence regions of the short-range correlated model Eq. (10) (dark shadow), the dashed lines those of the long-memory model with $\alpha=0.6$ (light shadow).

\section{Conclusions}

In this paper we studied the inference of long-range correlations by means of DFA with respect to the notions of sensitivity and specificity. We argue that the inference of a longrange correlated process underlying a given time series requires not only to show compatibility of the data with a longrange correlated process. Furthermore, other possible correlation structures, especially short-range correlations, have to be excluded.

Power-law scaling of the DFA fluctuation function is frequently taken as evidence for long-range correlations. To reliably infer power-law scaling, it must not be assumed but has to be established. This can be done by estimating local slopes and investigating them for constancy in a sufficient range. However, finite datasets bring along natural variability. To decide, if a fluctuating estimation of the slope has to be considered as being constant, we calculated empirical confidence intervals for a long-range and a simple shortrange correlated model. 
As a main insight, the inference of long-range correlations from a finite amount of data turned out to be not specific by means of DFA. Alternatively, one can investigate, if shortrange correlations can be inferred for the underlying process. For this setting, the specificity increases with the length of the data record.

Discussing typical difficulties of interpreting DFA results, we remark that scaling cannot be concluded from a straight line fit to the fluctuation function in a log-log representation. Additionally, we show that a local slope larger than $\alpha=0.5$ for large scales does not necessarily imply long-memory. If the length of the time series is not sufficiently large compared to the time scales involved, also for short-memory processes $\alpha=0.5$ may not be reached. Finally, we demonstrated, that it is not valid to conclude from a finite scaling region of the fluctuation function to an equivalent scaling region of the autocorrelation function.

Keeping these findings in mind, we reviewed the DFA results for the Prague daily air temperature record and compare them to a long-range and a short-range correlated model constructed using AR[1]-processes. Considering only large scales, for the given data set, one cannot reject the one or the other model. For the short-range correlated model, a much longer series would be necessary to identify a local slope of $\alpha=0.5$. The short-range correlated model we used involves time scales up to $\approx 1.5$ years. This might be regarded as long compared to the sampling time of one day but it is considerably small compared to the decades of persistence which are proposed in e.g. Koscielny-Bunde et al. (1998) and Govindan et al. (2002).

However, it is not our intention to advocate for ARprocesses being suitable to model temperature variation. For a certain range of scales, a power-law model can be formally suitable to describe characteristics of even short-memory processes. This, however, does not give evidence for longmemory.

Acknowledgements. This work was supported by the German Federal Ministry for Education, Science, Research and Technology under grant number 03330271 and by the Deutsche Forschungsgemeinschaft, Sonderforschungsbereich 555, project $\mathrm{C} 1$. We wish to thank J. Kurths for his encouragement and J. Kropp for inspiring discussions. The temperature data were provided by the Potsdam Institute of Climate Impact Research.

Edited by: M. Thiel

Reviewed by: two referees

\section{References}

Avnir, D., Birham, O., Lindar, D., and Malcai, O.: Is the Geometry of Nature Fractal, Science, 279, 39-40, 1998.

Beran, J.: Statistics for Long-Memory Processes, Monographs on Statistics and Applied Probability, Chapman \& Hall, 1994.

Beran, J., Bhansali, R., and Ocker, D.: On unified model selection for stationary and nonstationary short- and long-memory autoregressive processes, Biometrika, 85, 921-934, 1998.
Caputo, J. G., Malraison, B., and Atten, P.: Determination of attractor dimension and entropy for various flows: An experimentalist's viewpoint, in Dimensions and Entropies in Chaotic Systems, edited by Mayer-Kress, G., Springer Series in Synergetics, Springer Verlag, Berlin, 32, 1986.

Chen, Z., Ivanov, P., Hu, K., and Stanley, H.: Effect of nonstationarities on detrended fluctuation analysis, Phys. Rev. E, 65, 041107, $1-15,2002$.

Denker, M. and Keller, G.: Rigorous statistical procedures for data from dynamical systems, J. Stat. Phys., 44, 67-93, 1986.

Elek, P. and Márkus, L.: A long range dependent model with nonlinear innovations for simulating daily river flows, Nat. Haz. Earth Sys. Sci., 4, 277-283, 2004,

SRef-ID: 1684-9981/nhess/2004-4-277.

Fraedrich, K. and Blender, R.: Scaling of Atmosphere and Ocean Temperature Correlations in Observations and Climate Models, Phys. Rev. Lett., 90, 108501, 1-4, 2003.

Frisch, U.: Turbulence: The Legacy of A. N. Kolmogorov, Cambridge University Press, 1995.

Giratis, L., Kokoszka, P., and Leipus, R.: Testing for long memory in the presence of a general trend, J. Appl. Prob., 38, 1033-1054, 2001.

Govindan, R. B., Vyushin, D., Bunde, A., Brenner, S., Havlin, S., and Schellnhuber, H.-J.: Global Climate Models Violate Scaling of the Observed Atmospheric Variability, Phys. Rev. Lett., 89, 028501, 1-4, 2002.

Granger, C.: Long Memory Relationships and the Aggregation of dynamic models, J. of Econometrics, 14, 227-238, 1980.

Hasselmann, K.: Stochastic climate models: Part I. Theory, Tellus, 28, 473-485, 1976.

Hausdorff, J. M. and Peng, C.-K.: Multiscaled randomness: A possible source of 1/f noise in biology, Phys. Rev. E, 54, 2154-2157, 1996.

Honerkamp, J.: Statistical Physics, Springer, Berlin, 1998.

Hoskins, J.: Fractional Differencing, Biometrica, 68, 165-176, 1981.

Hu, K., Ivanov, P. C., Chen, Z., Carpena, P., and Stanley, H. E.: Effect of Trends on Detrended Fluctuation Analysis, Phys. Rev. E, 64, 011114, 1-19, 2001.

Kantelhardt, J. W., Koscielny-Bunde, E., Rego, H. H. A., Havlin, S., and Bunde, A.: Detecting Long-range Correlations with Detrended Fluctuation Analysis, Physica A, 295, 441-450, 2001.

Kantz, H. and Schreiber, T.: Dimension estimates and physiological data, Chaos, 5, 143-153, 1995.

Koscielny-Bunde, E., Bunde, A., Havlin, S., Roman, H. E., Goldreich, Y., and Schellnhuber, H.-J.: Indication of a Universal Persistence Law Governing Atmospheric Variability, Phys. Rev. Lett., 81, 729-732, 1998.

Mandelbrot, B. and van Ness, J. W.: Fractional Brownian motions, fractional noises and applications, SIAM Rev., 10, 422437, 1968.

McLeod, A. I. and Hipel, K. W.: Preservation of the Rescaled Adjusted Range. 1. A Reassessment of the Hurst Phenomenon, Wat. Resour. Res., 14, 491-508, 1978.

Montanari, A., Rosso, R., and Taqqu, M. S.: A seasonal fractional ARIMA model applied to the Nile River monthly flows, Wat. Resour. Res., 36, 1249-1259, 2000.

Pelletier, J. and Turcotte, D.: Self-Affine Time Series: II. Applications and Models, Advances in Geophysics, 40, 91-166, 1999.

Peng, C.-K., Buldyrev, S. V., Goldberger, A. L., Havlin, S., Simons, M., and Stanley, H. E.: Finite-size effects on long-range correlations: Implications for analyzing DNA sequences, Phys. Rev. E, 
47, 3730-3733, 1993.

Peng, C.-K., Buldyrev, S. V., Havlin, S., Simons, M., Stanley, H. E., and Goldberger, A. L.: Mosaic organization of DNA nucleotides, Phys. Rev. E, 49, 1685-1689, 1994.

Taqqu, M. S., Teverovsky, V., and Willinger, W.: Estimators for long-range dependence: An empirical study, Fractals, 3, 785798, 1995.

Timmer, J. and König, M.: On Generating Power Law Noise, A\&A, 300, 707-710, 1995.

Timmer, J., Haeussler, S., Lauk, M., and Luecking, C. H.: Pathological tremors: Deterministic chaos or nonlinear stochastic oscillators?, Chaos, 10, 278-288, 2000.
Trenberth, K.: Persistence of Daily Geopotential Heights over the Southern Hemisphere, Month.Wea.Rev., 113, 38-53, 1985.

Tsonis, A. and Elsner, J.: Testing for Scaling in Natural Forms and Observables, J. Stat. Phys., 81, 869-880, 1995.

Tsonis, A., Roeber, P., and Elsner, J.: On the existence of spatially uniform scaling laws in the climate system, in Paradigms of complexity, edited by Novak, M., World Scientific, Singapore, 2528,2000

von Storch, H. and Zwiers, F.: Statistical Analysis in Climate Research, chap. Specific Statistical Concepts in Climate Research, Cambridge University Press, Cambridge, 1999. 